BULL. AUSTRAL. MATH. SOC.

VOL. 31 (1985), 181-184.

\title{
THE POWER CONCAVITY OF SOLUTIONS OF SOME SEMILINEAR ELLIPTIC BOUNDARY-VALUE PROBLEMS
}

\section{GRANT KEADY}

Let $\Omega$ be a bounded convex domain in $R^{2}$ with a smooth boundary. Let $0<\gamma<1$. Let $u \in C^{2}(\Omega) \cap C(\bar{\Omega})$ be a solution, positive in $\Omega$, of

$$
\begin{aligned}
-\Delta u & =u^{Y} & \text { in } & \Omega, \\
u & =0 & \text { on } & \partial \Omega .
\end{aligned}
$$

Then the function $u^{\alpha}$ is concave for $\alpha=(1-\gamma) / 2$.

Let $\Omega$ be a bounded convex domain in $\mathbf{R}^{2}$ with a smooth boundary. To avoid some minor technicalities, assume that the curvature on $\partial \Omega$ is uniformly bounded away from zero. We give a new proof of the following theorem, using techniques which generalise those of Makar-Limanov [6] $(\gamma=0)$ and of Acker, Payne and Philipin [1] $(\gamma=1)$.

Let $u$ be any positive function on $\Omega$. The function $u$ is said to be $\alpha$-concave, for $\alpha>0$, if $u^{\alpha}$ is concave. The function $u$ is said to be 0 -concave, or $\log$-concave, if $\log u$ is concave.

THEOREM 1. Let $0 \leq \gamma \leq 1$. Let $u \in C^{2}(\Omega) \cap C(\bar{\Omega})$ be a solution, positive in $\Omega$, of

Received 11 September 1984.

Copyright Clearance Centre, Inc. Serial-fee code: 0004-9727/85 $\$ A 2.00+0.00$. 


$$
\left\{\begin{array}{c}
-\Delta u=u^{\gamma} \text { in } \Omega, \\
u=0 \text { on } \partial \Omega .
\end{array}\right.
$$

Then the function $u$ is a-concave for $0 \leq \alpha \leq(1-\gamma) / 2$.

Concerning the interior regularity needed in the proof, it is known that any solution $u \in C^{2}(\Omega)$ is in $C^{\infty}(\Omega)$.

Theorem I was first proved in a University of Adelaide PhD thesis by Kennington [4]. Kennington's proof actually establishes the theorem in $\mathbf{R}^{n}$ (with $n \geq 2$ ). Kennington's techniques are clever extensions of those of Korevaar [5]. Korevaar used his techniques to establish the result in the case $\gamma=1$. A similar proof, again for $\gamma=1$, appears in Caffarelli and Spruck [2].

The proof of Theorem 1 below is just one application of the Maximum Principle (Protter and Weinberger [7], Sperb [8]) in the following form.

MAXIMUM PRINCIPLE. Let $\theta \in C(\Omega)$ with $\theta \geq 0$. Let

$$
Z=\{z \in \Omega \mid \Theta(z)=0\} \text {. }
$$

Let $I \in C^{2}(\Omega)$ satisfy

$$
-\Delta I+\frac{A \cdot \nabla I}{\theta}+A_{0} I=0
$$

$$
I>0 \text { in a neighbourhood of } 2 \Omega \text {, }
$$

where $A$ and $A_{0}$ belong to $C(\Omega)$ with $A_{0} \geq 0$. Suppose that $I>0$ at points of $Z$. Then $I \geq 0$ in $\Omega$.

Note that the only use of the hypothesis on the curvature of $\partial \Omega$ is to guarantee (1.1). In the application to Problem (P), with $\gamma>0$, the boundary condition is

$$
I(z)++\infty \text { as } z \rightarrow \partial \Omega
$$

(Note also that the coefficient $A_{0}$ is singular at the boundary.)

The quantity $I$ is the most obvious generalisation of that used in [6] and [1], namely $I=I_{\alpha}$ with $\alpha=(1-\gamma) / 2$. Here, if $0<\alpha<1$, 


$$
I_{\alpha}=\frac{u^{2} \text { Hessian }\left(u^{\alpha}\right)}{\alpha^{2}(1-\alpha)}=\frac{u^{2}\left[\left(u^{\alpha}\right)_{x x}\left(u^{\alpha}\right)_{y y}-\left(\left(u^{\alpha}\right)_{x y}\right)^{2}\right)}{\alpha^{2}(1-\alpha)} .
$$

If $\alpha=0$,

$$
I_{0}=u^{2} \operatorname{Hessian}(\log u)
$$

For a positive superharmonic function $u$, establishing that $I_{\alpha} \geq 0$ in $\Omega$ is establishing that $u$ is $\alpha$-concave. (In the case $\gamma=0$ our notation is exactly as in [6], that is $I=I_{\frac{1}{2}}$. In the case $\gamma=1$ our $I=I_{0}$ is $\Phi / 2$ where $\Phi$ is defined by equation (2.1) of [1].)

Define

$$
P_{2}=|\nabla u|^{2}+\frac{2 u^{\gamma+1}}{1+\gamma}
$$

(The notation is that of Sperb [8].) The explicit formulae for the coefficients $A_{0}, A$ and $\theta$ are as follows:

$$
\begin{aligned}
A & =A_{1}+A_{2} \nabla I, \\
A_{2} & =-8 u^{1+\gamma}(1+\gamma), \\
A_{0} & =\frac{|\nabla u|^{2}}{u^{2}} \gamma(1-\gamma), \\
A_{1} & =2 \gamma(1+\gamma) \frac{P_{2}}{u}\left\{2 u \nabla P_{2}-(1+\gamma) P_{2} \nabla u\right\}
\end{aligned}
$$

and

$$
\begin{aligned}
\theta & =\left(4 u u_{x x}+2 u^{1+\gamma}-(1+\gamma)\left(u_{x}^{2}-u_{y}^{2}\right)\right)^{2}+4\left(2 u u_{x y}-(1+\gamma) u_{x} u_{y}\right)^{2} \\
& =-8 u^{1+\gamma}(1+\gamma) I+P_{2}^{2}(1+\gamma)^{2} .
\end{aligned}
$$

The form of the equation was discovered using the earlier results of [6] and [1] as a guide.

The coefficients were determined in the order, first $A_{2}$, then $A_{0}$ 
and finally $A_{1}$. The only important detail is the sign of $A_{0}$. Further details are given in the research report, Keady [3]. The calculations were sufficiently intricate that the computer algebra system, REDUCE, was used. The REDUCE programs are given in Keady [3].

\section{References}

[1] A. Acker, L.E. Payne and G. Philippin, "On the convexity of level lines of the fundamental mode in the clamped membrane problem, and the existence of convex solutions in a related free boundary problem", Z. Angew. Math. Phys. 32 (1981), 683-694.

[2] A. Caffarelli and J. Spruck, "Convexity properties of solutions to some classical variational problems", Comm. Partial Differential Equations 7 (1982), 1337-1379.

[3] Grant Keady, "The concavity of solutions of $-\Delta u=u^{\gamma} "$ (Research Report, 30. Centre for Mathematical Analysis, Australian National University, Canberra, Australia, 1984).

[4] Alan U. Kennington, "An improved convexity maximum principle and some applications" (PhD thesis, University of Adelaide, Adelaide, 1984). See also: Abstract, Bull. Austral. Math. Soc. 31 (1985), $159-160$.

[5] N.J. Korevaar, "Convex solutions to nonlinear elliptic and parabolic boundary value problems", Indiana Univ. Math. J. 32 (1983), $603-614$.

[6] L.G. Makar-Limanov, "Solution of Dirichlet's problem for the equation $\Delta u=-1$ in a convex region", Math. Notes 9 (1971), 52-53.

[7] H. Protter and H.F. Weinberger, Maximum principles in differential equations (Prentice Hall, Englewood Cliffs, New Jersey, 1967).

[8] R. Sperb, Maximum principles and their applications (Academic Press, New York, London, 1981).

Department of Mathematics, University of Western Australia, Nedlands, Western Australia 6009, Australia. 